\title{
Uso de Group Storytelling no Treinamento em Organizações
}

\author{
Aluno: Ricardo Tulio Gandelman \\ Orientadora: Flávia Maria Santoro \\ Programa de Pós-Graduação em Informática - Departamento de Informática Aplicada - \\ Universidade Federal do Estado do Rio de Janeiro (UNIRIO) - RJ - Brasil \\ \{flavia.santoro, ricardo.gandelman\} euniriotec.br
}

Nível: Mestrado; Ano de Ingresso: 2008; Previsão de Conclusão: Dezembro de 2009. Etapas já concluídas: Defesa da proposta

\begin{abstract}
Resumo. Treinamento em organizações é uma atividade importante para a sua sobrevivência. Treinamentos consomem recursos financeiros e tempo, e nem sempre podem ser feitos na prática reproduzindo casos reais. Isto tende a limitar a sua realização e por vezes, eles não atingem seus objetivos. Neste artigo apresenta-se uma proposta para projetos de treinamentos alinhados às necessidades das organizações, partindo de estórias reais, contadas por quem as vivenciou e, portanto, expressando a aplicação de conceitos na prática. Uma ferramenta de Group Storytelling com funcionalidades especificas é proposta nesta pesquisa.
\end{abstract}

Palavras-Chave: Aprendizagem Organizacional, Treinamento, Group storytelling 


\section{Introdução}

Treinamento é objeto de pesquisa em vários campos da ciência, pois é uma atividade importante tanto para a competitividade das organizações, como também para a motivação dos empregados [O'Brien e Hall 2004][Olfman, Bostrom e Sein 2003]. A velocidade da mudança na tecnologia e na competição exige das organizações maior atenção ao treinamento [LeRouge e Webb 2003]. Enormes quantias são gastas em treinamento, que é um dos métodos de maior poder de penetração para aumentar a produtividade dos indivíduos [Gupta e Bostrom 2006].

Vários trabalhos demonstram a relação direta entre treinamento e produtividade. Treinamentos corrigem deficiências e permitem aos empregados enfrentar desafios no desempenho de suas tarefas, além de motivarem equipes e servirem como catalisadores para a ação [Li, Yang e Lin 2008][Simonsen e Sein 2004]. No entanto, há diversas barreiras impostas pelo treinamento tradicional às pequenas e médias empresas: falta de tempo dos profissionais que, por serem em número reduzido não podem afastar-se de suas funções; o custo de treinamentos, especialmente quando o número de pessoas a treinar é grande; a não adequação dos cursos às necessidades reais das empresas [O'Brien e Hall 2004].

O treinamento tradicional não mais consegue atender às necessidades atuais de educação continuada, centrada na gestão de competências e resultados [Silveira 2007]. Para isto, é necessário um novo processo educacional nas organizações, apoiado por um sistema que garanta o aprendizado de técnicas e metodologias modernas, focadas nas estratégias de negócio [Costa 2001, Eboli 2002 e Meister 1999 apud Silveira 2007]. Quando uma organização decide adotar um programa de treinamento, independente do objetivo, este deve estar voltado para o seu negócio [Carvalho 2002 apud Silveira 2007].

$\mathrm{O}$ problema tratado neste trabalho é como oferecer treinamentos orientados às necessidades da organização e de seus indivíduos. A proposta se baseia no uso sistemas computacionais de apoio ao Group Storytelling [Leal et al. 2004], através dos quais profissionais produzem narrativas para gerar cenários de treinamento alinhados com os processos de negócio das organizações.

\section{Trabalhos Relacionados}

Algumas abordagens foram identificadas na literatura como soluções para os problemas do treinamento tradicional: (i) baseados em Realidade Virtual (RV), (ii) baseados em elearning, (iii) baseados em jogos e simulação. Estas abordagens são detalhadas a seguir.

Mollet e Arnaldi (2006) propõem uma plataforma de autoria para a construção de aplicações de treinamento com realidade virtual. A plataforma é composta de quatro mecanismos: (i) ambiente para criação de um mundo 3D; (ii) motor para gerenciamento da interação entre os objetos; (iii) motor pedagógico para supervisionar as ações dos treinandos; (iv) motor de cenários para descrever o que pode ser feito em cada momento, oferecendo o próximo passo a ser executado, ou executando procedimentos. Elaborar cenários em RV não é trivial, assim como modelar seus objetos, e implementar o mecanismo didático adequado. Nota-se que as propostas nesta linha não contemplam a fonte para criação dos cenários, sendo muito mais focadas em infra-estrutura. 
Vários autores apontam o uso do e-learning como ferramenta de educação corporativa [Oliveira e Francisco 2005][Ikeda e Cavalheiro 2005]. Silveira e Filho (2007) afirmam que o e-learning pode ser utilizado para treinamento "quando é preciso moldar o aprendizado para um determinado objetivo". É voltado para empresas com grande número de funcionários, utilizando auto-estudo linear em conjunto com exercícios para fixação e tutoria. Silveira e Filho (2007) citam pesquisa realizada em 2006, com empresas de vários setores, que concluiu que elas não produzem seus elearnings, mas contratam cursos em várias consultorias. Parte-se do princípio que o conhecimento, na maioria das vezes, não está disponível nas empresas.

Lopes e Wilhelm (2006) e Carvalho, Haguenauer et al (2005) analisam o uso de jogos de simulação empresarial para apoiar o processo de ensino-aprendizagem e os consideram mais efetivos que os treinamentos tradicionais. A aplicação de jogos exige ferramentas e processos, interface e regras, preparação do ambiente, planejamento da atividade, formação das equipes, relação teoria-prática, interação inter-equipe, relação com as outras equipes, foco na aprendizagem e procedimentos de avaliação. Isto implica em grandes desafios de planejamento e desenvolvimento dos ambientes.

De maneira geral, as propostas para treinamento não consideram conteúdo advindo das práticas internas das organizações: a RV está mais preocupada com objetos e cenários, e não com a fonte destes cenários, que seriam as práticas e processos; o conteúdo de e-learning não é produzido nas organizações, mas por consultorias externas que desconhecem as práticas internas; os jogos são voltados para situações que focam a organização como um todo, simulando departamentos, e não práticas e processos.

A proposta deste trabalho pretende resolver os problemas dos treinamentos tradicionais, e adicionalmente garantir a adequação do treinamento às necessidades da organização, e seu alinhamento com seus processos de negócio.

\section{Fundamentação Teórica: Narrativas, Estórias e Group Storytelling}

Reissner (2005) e Senge (1990) afirmam que para muitas organizações, a meta final é tornar-se uma organização que aprende, na qual a aprendizagem é incorporada nas rotinas diárias. Para isto, as novas idéias precisam ser agregadas ao modelo mental dos empregados. O uso de estórias em um contexto específico permite que se construam significados e que realidades sejam construídas. "O aprendizado é uma conseqüência do conceito de fazer sentido" [Weick 1995 apud Reissner 2005]. Desta forma, estórias servem como ferramenta de aprendizagem.

Storytelling é "um meio tradicional e antigo de passar sabedoria e cultura" [Sole e Wilson 2002 apud Tobin e Snyman 2008]. No contexto das organizações, estórias são seqüências de decisões, ações ou eventos, que envolvem personagens, nas quais um desafio de negócio ou uma oportunidade precisam ser enfrentados. Storytelling pode ser definido como "práticas, ferramentas e interpretação de papéis envolvidos na comunicação de estórias para a audiência" [Tobin e Snyman 2008].

Leal et al. (2004) propõem que estórias sejam contadas de forma coletiva, incorporando várias perspectivas com descrições ou alternativas aos mesmos fatos. $\mathrm{O}$ conceito básico na narração em grupo ou Group Storytelling é que uma pessoa recorda parcelas de conhecimento, que podem ser complementadas com o conhecimento de 
outros participantes, pois leituras e comentários ativam a memória, aumentando a habilidade de uma pessoa de contar algo que testemunhou. As estórias escritas por uma equipe de trabalho encerram detalhes valiosos e cada participante tem a oportunidade de interagir e apresentar sua percepção sobre os fatos [Santoro e Brezillon 2006].

Há inúmeros benefícios no uso das estórias e de group storytelling: ajuda a fixar conceitos de forma mais permanente; é uma prática instrucional efetiva que permite às pessoas entenderem as coisas de maneira relevante e que façam sentido para elas; é um "meio de comunicação rápido, natural claro, confiável, colaborativo, persuasivo, acurado, intuitivo, divertido, comovente e interativo"; permite a comunicação de idéias complexas de forma simples, possibilitam razão e emoção [Tobin e Snyman, 2008].

O'Brien e Hall (2004) afirmam que os treinamentos online estão a cada dia mais difundidos, porém as pequenas e médias empresas não têm conseguido beneficiar-se deles, devido a barreiras técnicas, organizacionais e culturais, como também pela insuficiência dos recursos, já que os sistemas comerciais existentes são voltados ao mercado de massa. Eles propõem que as próprias empresas construam seus treinamentos online, de acordo com seus interesses e necessidades. O uso de Group Storytelling é uma das maneiras de concretizar tais treinamentos.

\section{A Proposta: Uso de Group Storytelling para Projeto de Treinamento}

O elemento central da proposta é a narrativa. Narrativas que contenham cenários, personagens e uma situação, vivenciados por pessoas reais ou imaginárias, a partir de situações reais ou imaginárias da organização, para as quais seja necessário treinar os indivíduos. $\mathrm{O}$ método proposto é baseado em narrativas utilizando suporte computacional, onde estórias são as fontes para a criação de desenhos animados interativos que retratam situações reais ou fictícias. As estórias serão tratadas pedagogicamente, de forma que os desenhos tenham conteúdo educacional e instrutivo. As estórias, contextualizadas e originadas de problemas ou situações reais da organização, irão assegurar que os treinamentos estejam de acordo com as necessidades da organização, e alinhados aos seus processos de negócio.

Uma ferramenta de suporte ao método deverá permitir a criação, classificação, armazenamento e recuperação de estórias, em função das solicitações dos indivíduos, para a busca do treinamento adequado à sua situação. Deve estar disponível para acesso web, de forma que possa ser utilizada a qualquer tempo e de qualquer local. Além disso, a ferramenta deverá incorporar funcionalidades para geração das animações. Para isso será analisado o LOGTELL [Ciarlini, Pozzer et al. 2005], que é uma ferramenta para autoria interativa e dramatização de estórias. Experimentos utilizando tal ferramenta mostraram que ela pode ser usada para criar e adaptar estórias para outros fins além de entretenimento. LOGTELL provê módulos para a geração, gerenciamento e visualização de tramas interativas, com uso de animação 3D em tempo real dramatizada por atores virtuais, aonde o autor intervém na geração da trama, conduzindo o processo criativo.

Spaniol et al. (2006) citam ferramentas de storytelling, como "Dramatica", "Adaptive Digital Storytelling (Adaptive DST)", "Storylining Suspense \& Story Engine", "Hypermidia Novel" e "Digital Storytelling Cookbook and Travelling 
Companion (DSC)" e "Movement Oriented Design (MOD)", além de sua própria proposta, "Media Integrated Story-Telling (MIST)", que serão também analisadas.

\section{Situação atual da pesquisa}

A proposta baseia-se em ferramenta de storytelling, com funcionalidades adicionais para geração de treinamentos animados. Uma seleção de ferramentas de autoria/ambiente de storytelling será feita segundo critérios a definir. Algumas características, porém, já pode ser adiantadas: (i) permitir a autoria de estórias; (ii) permitir a classificação e recuperação de estórias, baseadas em definições fornecidas pelo autor; (iii) interface que permita buscar, recuperar e analisar informações, documentos, páginas ou outros artefatos pertinentes, a partir das ferramentas colaborativas utilizadas na organização (wikis, blogs, fóruns etc.); (iv) mecanismo que permita construir e armazenar relações entre as estórias e os artefatos descritos no item anterior; (v) acessível através da web.

Após a análise das ferramentas, a selecionada será modificada para atender às necessidades específicas citadas acima. Será feito um estudo de caso, focado em um contexto pré-determinado, para avaliar em que medida a ferramenta permite que os treinamentos sejam, de fato, alinhados aos processos de negócio da organização. Questionário e entrevistas serão utilizados para fornecer dados para a avaliação.

\section{Conclusão}

Pesquisas apontam que treinamentos baseados em suporte computacional, como ambientes de realidade virtual, e-learning, jogos e simulação, e storytelling apresentam algumas vantagens sobre os treinamentos tradicionais. A proposta aqui apresentada trata da questão da relevância de conteúdo, já que ele é baseado nos acontecimentos reais da organização, ou preparado especificamente para tal através estórias contextualizadas.

Outras importantes vantagens em relação aos trabalhos relacionados: permitir que o conteúdo seja tão granular quanto se queira, garantindo uma contextualização maior; customização dos treinamentos, uma vez que as estórias são pontuais e tratam de situações específicas e em contextos específicos; a disponibilidade via web garante a disponibilidade e acessibilidade, sem restrições de local ou tempo. Como todo o conteúdo é produzido baseado em problemas, situações, ocorrências, processos e atividades da organização, não só a estória como os artefatos a ela associados, a proposta garante o alinhamento aos processos de negócio da organização.

\section{Referências}

Carvalho, F.S., Haguenauer, C.J. e Victorino, A.L.Q. (2005), "Utilização de Jogos Interativos no Ensino a Distância Via Internet", Congresso Internacional de Educação à Distância, Florianópolis, Brasil.

Ciarlini, A.E.M., Pozzer, C.T., Furtado, A.L., Feijó, B. (2005), “A logic-based tool for interactive generation and dramatization of stories". International Conference on Advances in Computer Entertainment Technology, New York, USA, p. 133-140.

Gupta, S. e Bostrom, R. P. (2006), "End-user training methods: what we know, need to know", Conf. on Computer Personnel Research", New York, USA, p. 172-182.

Ikeda, A.A. e Cavalheiro, C. (2005), "Reflexões sobre as Contribuições do Ensino a 
Distância", eGesta Revista Eletrônica de Gestão de Negócios, v. 1, n. 3, out-dez 2005, p. 55-75, Universidade Católica de Santos.

Leal, R.P.; Borges, M.R.S., Santoro, F.M. (2004). ”Applying Group Storytelling in Knowledge Management”, Lecture Notes in Computer Science, v. 3198, p. 34-41.

LeRouge, C., Webb, H. (2003), "Managing Training in a Technology Context", Conference on Computer Personnel Research, ACM, New York, USA, p. 98-103.

Li, C.-M., Yang, T.L. e Lin, P.C. (2008), “Training Program for R\&D Staff”, Int. Conference on Management of Engineering \& Technology, 2008, p. 1466-1469.

Lopes, M. C. e Wilhelm, P. P. H. (2006), "Uso de Jogos de Simulação Empresarial como Ferramenta Educacional: Uma Análise Metodológica", Revista Tecnologia da Informação, v. 6, n. 2, p.19-29.

Mollet, N.e Arnaldi, B. (2006), "Storytelling in Virtual Reality for Training", Pan, Z.G., Diener, H., Jin, X.G., Gobel, S. e Li, L. Ed., Technologies for E-Learning and Digital Entertainment, Proceedings, Springer-Verlag Berlin, Germany, p. 334-347.

O'Brien, E., Hall, T. (2004), "Training Needs Analysis: the First Step in Authoring eLearning Content”, Symp.on Applied Computing, New York, USA, p. 935-939.

Olfman, L., Bostrom, R.P., Sein, M.K. (2003), "A Best-Practice Based Model for Information Technology Learning Strategy Formulation”, Proceedings of SIGMIS Conference on Computer Personnel Research”, ACM, New York, USA, p. 75-86.

Oliveira, A.C., Francisco, A. C. (2005), "Educação Corporativa Baseada em e-Learning - Uma Ferramenta para a Gestão do Conhecimento Empresarial”, I Encontro Estadual de Engenharia da Produção e I Simpósio de Gestão Industrial, Ponta Grossa, Brasil.

Reissner, S. C. (2005), "Learning and Innovation: a Narrative Analysis", Journal of Organizational Change Management, v. 18, n. 5, p. 482-494.

Santoro, F.M.; Brezillon, P. (2006), "The Role of Shared Context in Group Storytelling”, Computing and Informatics, v. 25, p. 1001-1026.

Senge, P. (1990) “A quinta disciplina”, Ed. Best Sellers, São Paulo.

Silveira, C. V. S., Filho, L. C. R. (2007), "Educação Corporativa: a Contribuição do eLearning para a Disseminação do Conhecimento Corporativo", UNIFIEO - Revista da Pós-Graduação, v. 1, n. 2, p. 88-94.

Silveira, V. N. S. (2007),'’Maturidade em Gestão de Pessoas e Práticas de Treinamento, Desenvolvimento e Educação: Uma análise a partir do People Capability Maturity Model (P-CMM)”, Dissertação de Mestrado, UFMG, Belo Horizonte, Brasil.

Simonsen, M. e Sein, M. K. (2004), "Conceptual Frameworks in Practice: Evaluating End-User Training Strategy in an Organization", Proceedings of the 2004 SIGMIS Conference on Computer Personnel Research", ACM, New York, USA, p. 14-24.

Spaniol, M., Klamma, R., Sharda, N. e Jarke, M. (2006), 'Web-Based Learning with Non-linear Multimedia Stories', Advances in Web Based Learning, p 249-263.

Tobin, P. K. J. e Snyman, R. (2008), "Once Upon a Time in Africa: a Case Study of Storytelling for Knowledge Sharing”, in Aslib Proceedings, v. 60, n. 2, p.130-142. 